\title{
Erratum
}

Notfall Rettungsmed 2020 23:660 https://doi.org/10.1007/s10049-020-00797-2 Online publiziert: 20. Oktober 2020 (c) Springer Medizin Verlag GmbH, ein Teil von Springer Nature 2020

Michael Dommasch ${ }^{1}$ Friedemann Gebhardt ${ }^{2} \cdot$ Ulrike Protzer $^{3} \cdot$ Angelika Werner $^{4}$. Eva Schuster ${ }^{5}$. Christoph Brakemeier ${ }^{6}$. Julia Mayer ${ }^{6}$. Susanne Feihl ${ }^{7}$. Christiane Querbach ${ }^{8} \cdot$ Rickmer Braren $^{9} \cdot$ Matthias Treiber $^{10} \cdot$ Fabian Geisler $^{10}$. Christoph D. Spinner"11

'Fakultät für Medizin, Zentrale Interdisziplinäre Notaufnahme, Klinikum rechts der Isar, Technische Universität München, München, Deutschland; ${ }^{2}$ Fakultät für Medizin, Krankenhaushygiene, Klinikum rechts der Isar, Technische Universität München, München, Deutschland; ${ }^{3}$ Fakultät für Medizin, Institut für Virologie, Klinikum rechts der Isar, Technische Universität München, München, Deutschland; ${ }^{4}$ Fakultät für Medizin, Stabsstelle für Qualitäts-und Risikomanagement, Klinikum rechts der Isar, Technische Universität München, München, Deutschland; ${ }^{5}$ Fakultät für Medizin, Unternehmenskommunikation, Klinikum rechts der Isar, Technische Universität München, München, Deutschland; ${ }^{6}$ Fakultät für Medizin, Pflegedirektion, Klinikum rechts der Isar, Technische Universität München, München, Deutschland; ${ }^{7}$ Fakultät für Medizin, Institut für medizinische Mikrobiologie, Immunologie und Hygiene, Klinikum rechts der Isar, Technische Universität München, München, Deutschland; ${ }^{8}$ Fakultät für Medizin,

Krankenhausapotheke, Klinikum rechts der Isar, Technische Universität München, München, Deutschland; ${ }^{9}$ Fakultät für Medizin, Institut für diagnostische und interventionelle Radiologie, Klinikum rechts der Isar, Technische Universität München, München, Deutschland; ${ }^{10}$ Fakultät für Medizin, Klinik und Poliklinik für Innere Medizin II, Klinikum rechts der Isar, Technische Universität München, München, Deutschland; "Fakultät für Medizin, Klinik und Poliklinik für Innere Medizin II (Infektiologie), Klinikum rechts der Isar, Technische Universität München, München, Deutschland

\section{Erratum zu: Strategie einer universitären Notaufnahme für das COVID-19-Management im Rahmen einer beginnenden Epidemie}

Erratum zu:

Notfall Rettungsmed 2020

https://doi.org/10.1007/s10049-

020-00759-8

In dem ursprünglichen Artikel wurde der Name der Autorin Christiane Querbach falsch geschrieben. Bitte beachten Sie die korrigierte Schreibweise.

\section{Korrespondenzadresse}

\section{Dr. med. Michael Dommasch}

Fakultät für Medizin, Zentrale Interdisziplinäre Notaufnahme, Klinikum rechts der Isar,

Technische Universität München Ismaninger Str. 22, 81675 München, Deutschland michael.dommasch@mri.tum.de
Die Online-Version des Originalartikels ist unter https://doi.org/10.1007/s10049-020-00759-8 zu finden. 